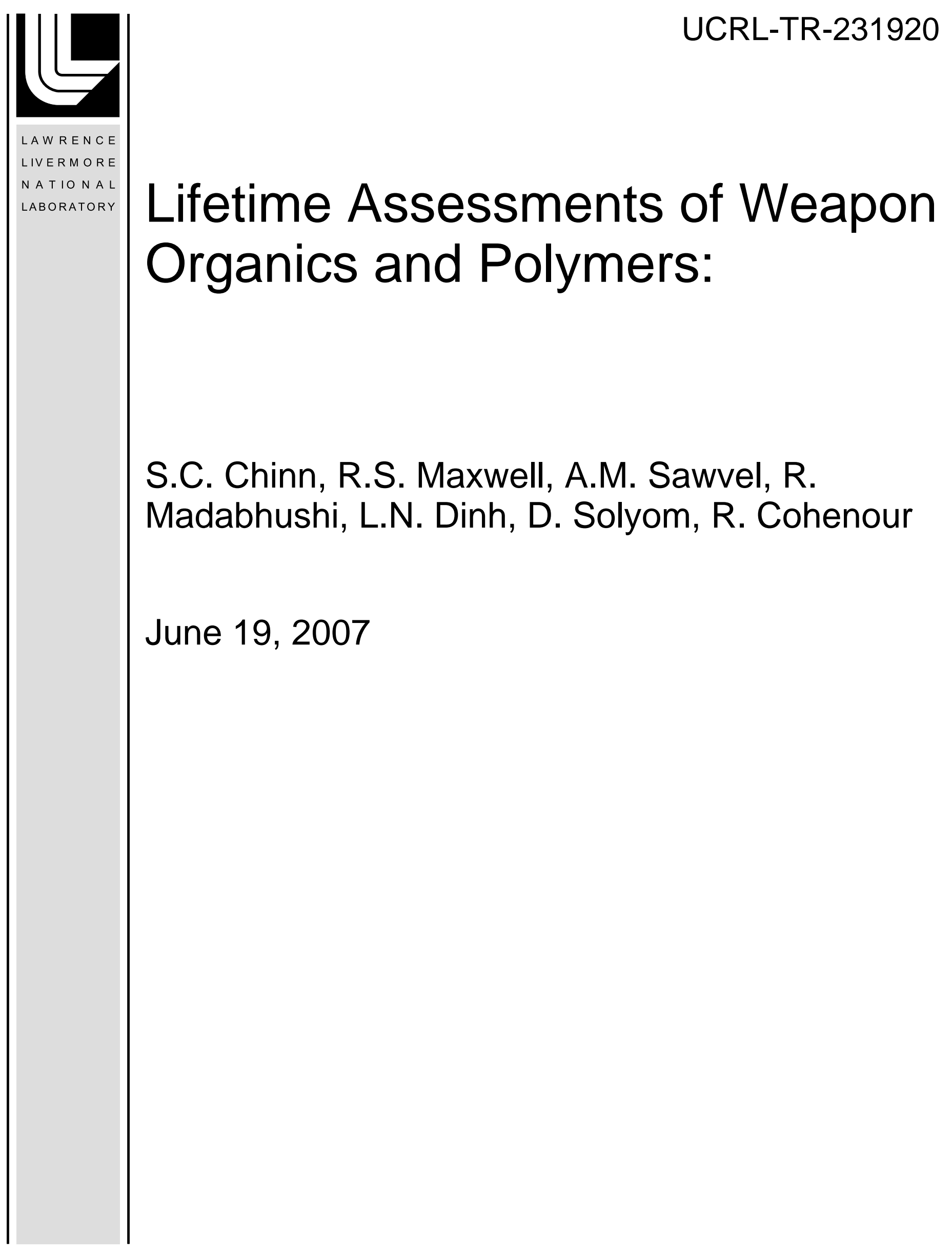


This document was prepared as an account of work sponsored by an agency of the United States Government. Neither the United States Government nor the University of California nor any of their employees, makes any warranty, express or implied, or assumes any legal liability or responsibility for the accuracy, completeness, or usefulness of any information, apparatus, product, or process disclosed, or represents that its use would not infringe privately owned rights. Reference herein to any specific commercial product, process, or service by trade name, trademark, manufacturer, or otherwise, does not necessarily constitute or imply its endorsement, recommendation, or favoring by the United States Government or the University of California. The views and opinions of authors expressed herein do not necessarily state or reflect those of the United States Government or the University of California, and shall not be used for advertising or product endorsement purposes.

This work was performed under the auspices of the U.S. Department of Energy by University of California, Lawrence Livermore National Laboratory under Contract W-7405-Eng-48. 


\title{
Lifetime Assessments of Weapon Organics and Polymers: FY05 Annual Report
}

\author{
Sarah C. Chinn, Robert S. Maxwell, April M. Sawvel, Ramki Madabhushi, Long N. Dinh \\ Lawrence Livermore National Laboratory \\ David Solyom, Rebecca Cohenour \\ Kansas City Plant
}

\begin{abstract}
Non-nuclear organics, while not yet regarded to be at risk, figure in a large percentage of Significant Findings Investigations. Further, early application of advanced lifetime, compatibility, and base-lining assessments for LEP replacement materials and emerging materials for the Reliable Replacement Warhead (RRW) provides data for rational material choices. This task is currently assessing the chemical and mechanical characteristics of a variety of weapon organics prioritized by the risk-consequence of material aging. The majority of effort to date has been dedicated to silicone elastomer components known to lose their load resistance with age, with additional effort dedicated to urethane adhesives. This fiscal year, we have focused on initial aging studies of UNI620-3, a candidate replacement material for DC745U, and Adiprene (LW520). Aging studies of S5455, originally scheduled for testing in FY05, were postponed until FY07. We have initiated aging studies on these materials by employing accelerated aging tests with exposure to Co-60 $\gamma$-radiation and elevated temperatures and studying their mechanical properties using a variety of analytical tools including nuclear magnetic resonance (NMR), dynamic mechanical analysis (DMA), and differential scanning calorimetry (DSC). Additionally, we have investigated aging signatures present in parts returned from surveillance as well as initiated long and short term stress relaxation studies for a thorough understanding of compression set occurring in silicone elastomers components. Additional investigations of aging processes in fluoropolymer binders and the combined effects of radiation and tensile set on silicone pads were performed. Finally, we have used temperature programmed desorption (TPD) studies to significantly improve the accuracy of our $\mathrm{H}_{2} \mathrm{O}$ outgassing model in comparison with last year through the use of the isoconversion technique as well as to measure the equilibrium vapor pressure of hydrogenated DPB pellets. This report satisfies the milestones/deliverables for the L3 task "Update chemical and mechanical aging models for cushion/pads/adhesives" and all data will be input into the L2 milestone "Bill's token L2 milesone."
\end{abstract}

\subsection{Introduction:}

Silicon based elastomers have, in general, the following advantageous characteristics: a wide service temperature range, excellent non-stick, non-adhesive properties; low toxicity; possible optical transparency; low chemical reactivity; and excellent resistance to attack by oxygen, ozone, and sunlight. When selecting a particular formulation for engineering applications, however, it is also important to recognize and identify the principle factors involved in degradation for prevention and stabilization efforts in material design issues. For example, the main structural modifications seen in irradiated polymers are changes in molecular weight distribution (due to main-chain scission, crosslinking, and endlinking) and the production of 
volatile degradation products [1]. It has further been observed that aging in dry environments can have dramatic effects on the mechanical properties of filled siloxane systems [2,3].

In this paper, we report on our continuing investigations into the effects of some degradation pathways on the chemical, microstructural, and polymer chain mobility properties of a family of silica-reinforced polysiloxane polymers. We have applied solid-state nuclear magnetic resonance spectroscopy (SS-NMR), Dynamic mechanical analysis (DMA), and differential scanning calorimetry (DSC) to assess changes in polymer structure, crosslink density, polymer-filler interactions, and phase behaviour. Temperature programmed desorption (TPD) has been used to further develop water offgassing kinetic models for certain materials. Each of these analytical methods identifies different aspects of the degradation mechanism, and when combined they provide a more complete understanding of the radiation-induced degradation process. This data is being interpreted in context of existing historical and surveillance data.

\subsection{Experiments:}

Nuclear magnetic resonance (NMR) (both high field and low field minispec and NMR MOUSE), differential scanning calorimetry (DSC), dynamic mechanical analysis (DMA), and temperature programmed desorption (TPD) have been applied to assess aging mechanisms in UNI620-3, LW520 (adiprene), M9747, S5370, and TR55. Detailed descriptions of the experimental methods as well as thermal and radiative aging methods can be found elsewhere [48].

\subsection{Results and Discussion:}

\subsection{Initial aging model of UNI620-3}

UNI620-3 is a candidate replacement material for DC745. Though the formulation is proprietary, it is believed a blend of two gums, one of which contains phenyl siloxane monomers. The addition of phenyl groups is well known to hinder the development of crystallinity in silicone elastomers [9]. More details of this material can be found in reference [10].

The siloxanes were exposed to thermal degradation at varying temperatures and times to investigate the effects of long-term exposure to high temperatures. The material was subject to extreme temperatures up to $300^{\circ} \mathrm{C}$ for up to 8 hours. NMR analysis using the Bruker minispec revealed relative changes in crosslink density as measured by the $T_{2}$ relaxation time. The data,
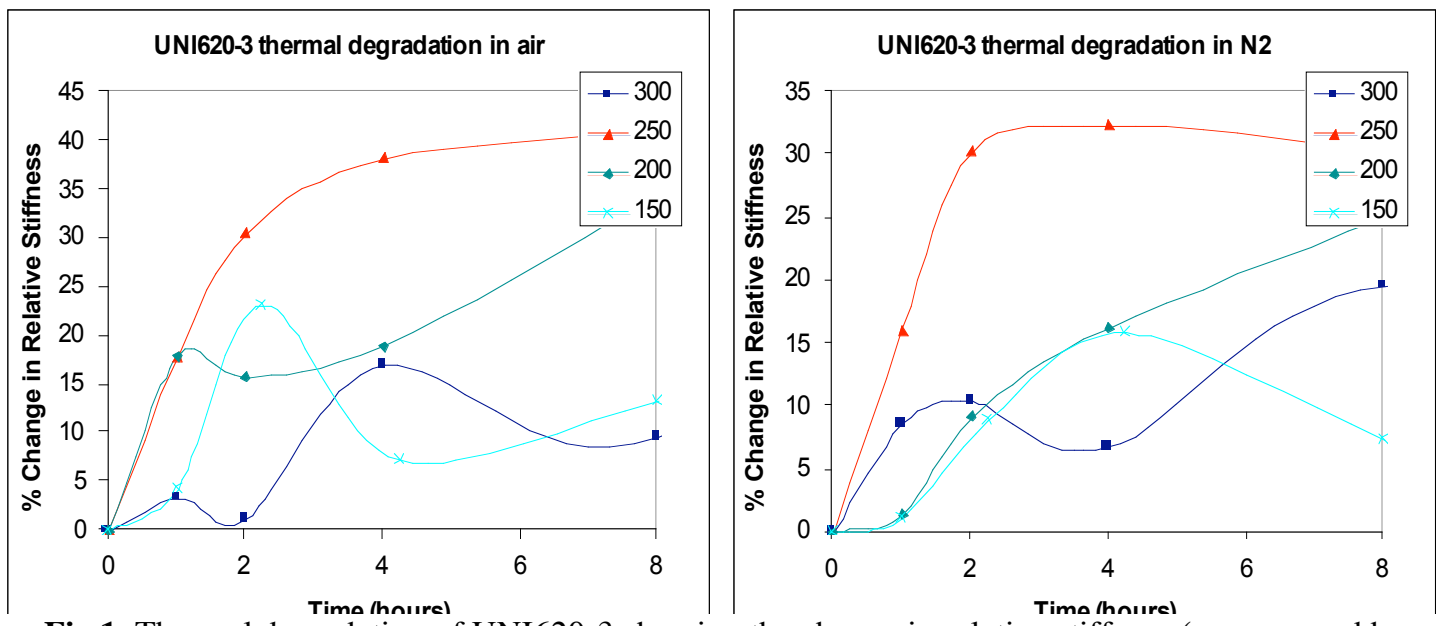

Fig 1. Thermal degradation of UNI620-3 showing the change in relative stiffness (as measured by NMR) as a function of time at different temperatures. 

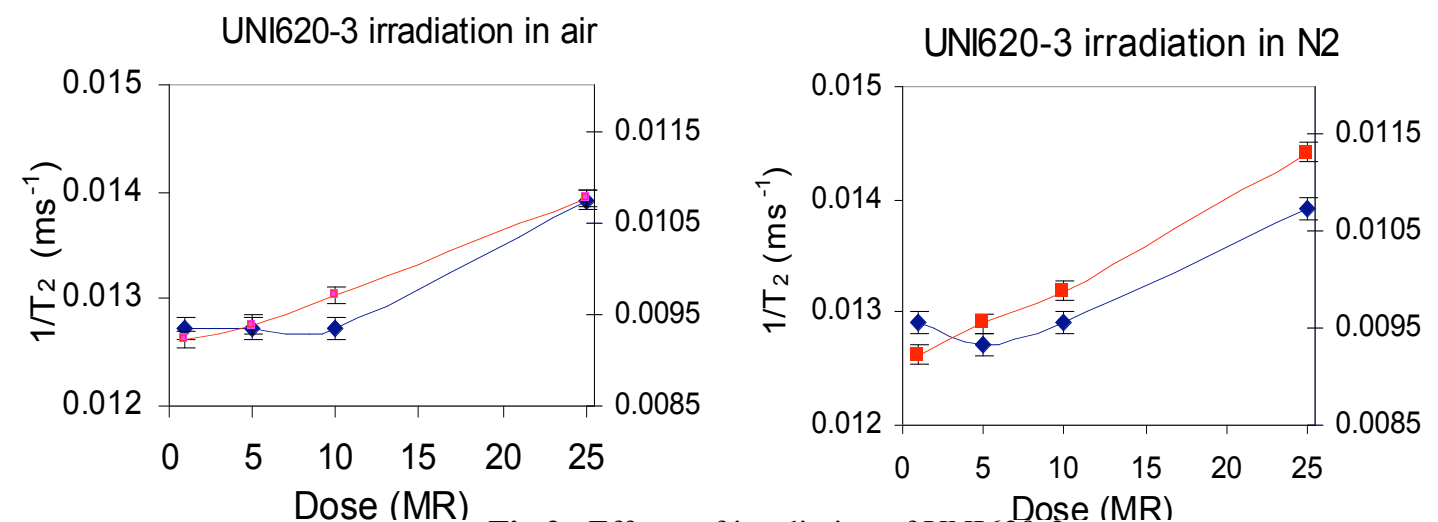

Fig 2. Effects of irradiation of UNI620-3.

shown in Figure 1, demonstrate an increase in stiffness with increasing temperatures up to $250^{\circ} \mathrm{C}$, while at $300^{\circ} \mathrm{C}$ the change in stiffness is not as extreme. Additionally, there was considerable fluctuation in the data with the exception of the sample aged at $250^{\circ} \mathrm{C}$, in which the relative stiffness increases steadily for the first four hours of aging before stabilization. This suggests there post curing is occurring as well as volatilization of the plasticizer. It is suggested that a more efficient post curing process is put in place if this material is to be used in service.

Samples were also exposed to high levels of Co-60 radiation to investigate their resistance to radiation. As shown in Figure 2, the material appears to be extremely resistant to radiation until $25 \mathrm{MR}$ of exposure. This increased resistance compared to DC745 is likely due to the increase in phenyl content of the UNI material. Though DSC and DMA results are pending,
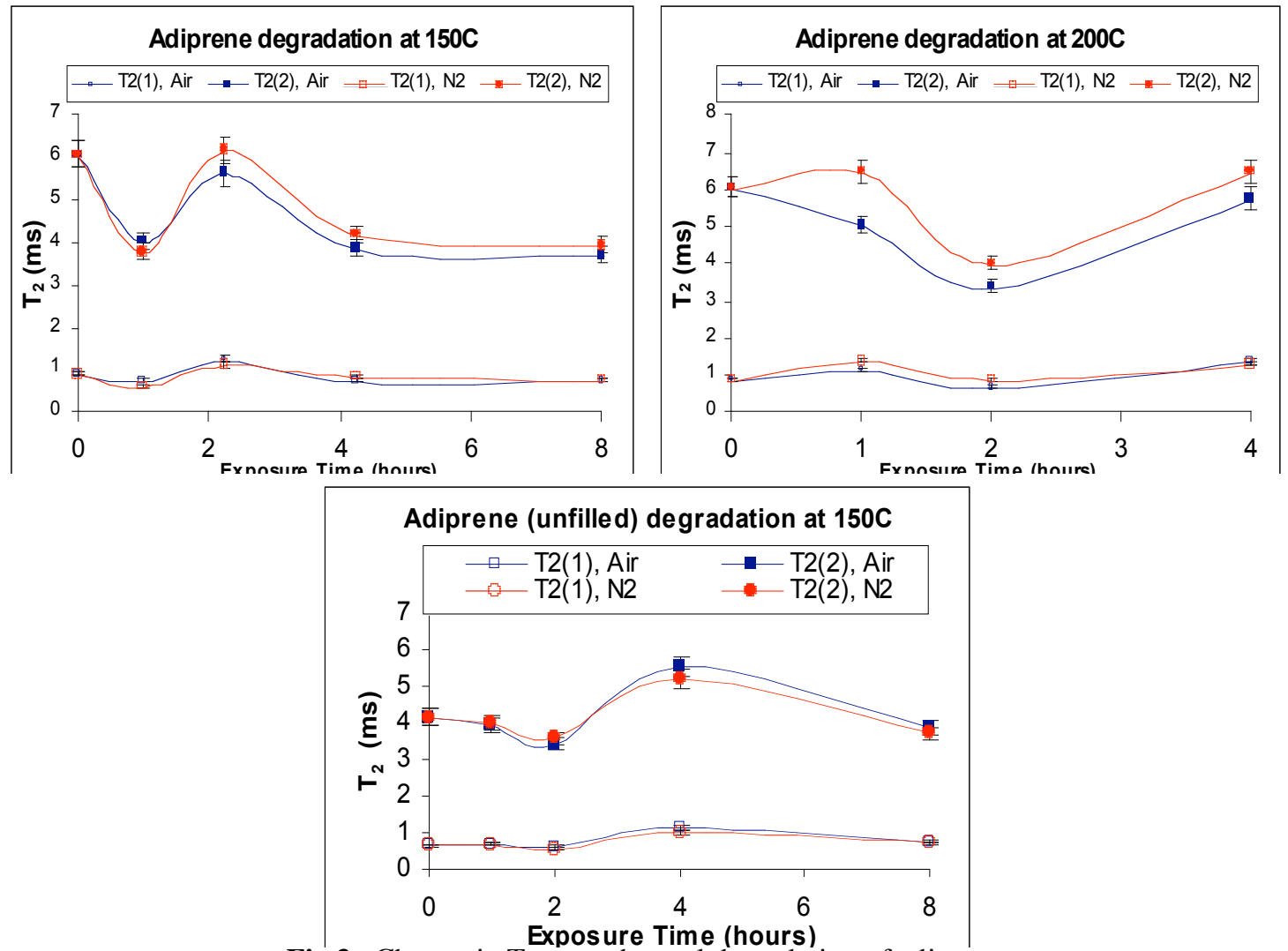

Fig 3. Change in $\mathrm{T}_{2}$ upon thermal degradation of adiprene. 

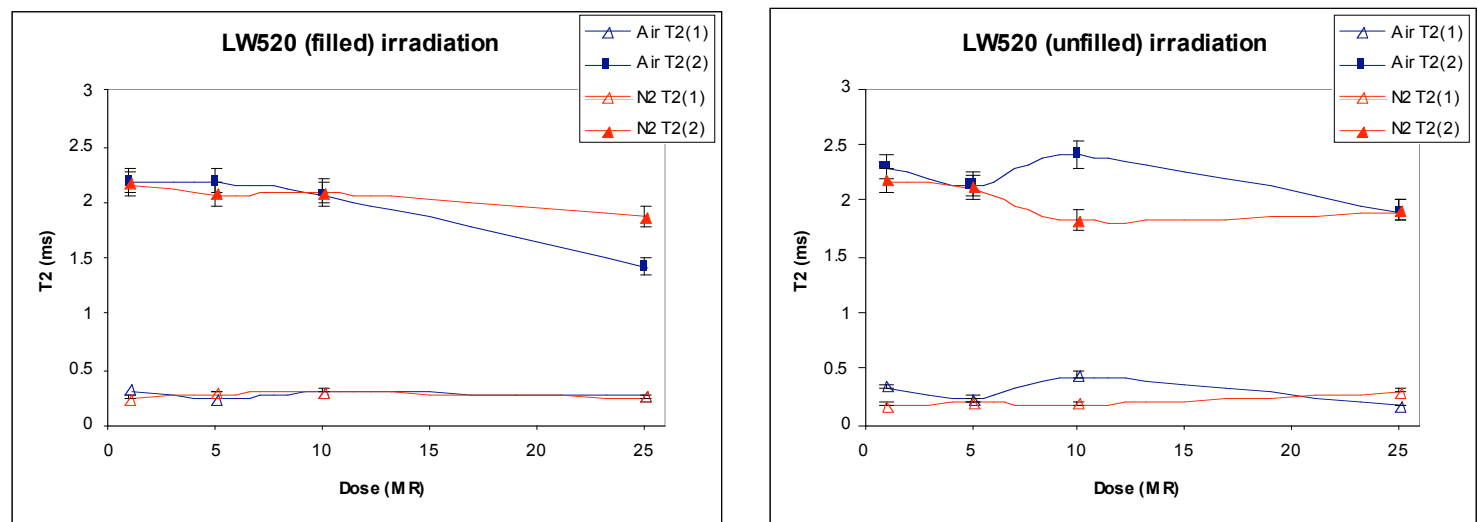

Fig 4. Change in $\mathrm{T}_{2}$ with radiation in filled and unfilled adiprene.

it can be assumed from the NMR results that radiation induced crosslinking is not expected to occur at stockpile relevant conditions.

\subsection{Initial aging model of Adiprene}

Adiprene is a polyurethane-based adhesive consisting of LW520 and XU-205. The material was prepared with and without Cab-o-Sil filler and cured at room temperature. Details about this polymer can be found in reference [11].

Thermal degradation was performed on filled and unfilled adiprene samples. The filled samples were visibly charred at $250^{\circ} \mathrm{C}$ and completely melted at $300^{\circ} \mathrm{C}$, while the unfilled samples were melted after exposure to $200^{\circ} \mathrm{C}$ for one hour. At lower temperatures, the $\mathrm{T}_{2}$ relaxation time, which is an indication of crosslink density [4], fluctuated at shorter exposure times and in the sample aged at $150^{\circ} \mathrm{C}$, there was no change observed between 4 and 8 hours of exposure in the filled sample. In the unfilled sample, the $\mathrm{T}_{2}$ increased after four hours of exposure, indicating a softening of the polymer possibly due to chain scission. It is evident that the Cab-o-Sil filler provides an increased resistance to thermally-induced chain scission that typically causes a softening and eventual melting in the polymer.

Adiprene was also exposed to various doses of Co-60 radiation. The NMR data, shown in Fig 4, shows that minimal changes in crosslink density occurred with doses up to $25 \mathrm{MR}$ with the exception of filled adiprene irradiated in an oxygenrich environment. Additional high resolution multiple quantum experiments were performed which revealed similar results, namely that the 25 MR (air) sample displayed increased residual dipolar couplings between the two different methylene components of the polymer [12].

Initial DSC analysis (not shown here) showed a glass transition around $75^{\circ} \mathrm{C}$ but additional thermal events did not appear in any samples. In Figure 5,

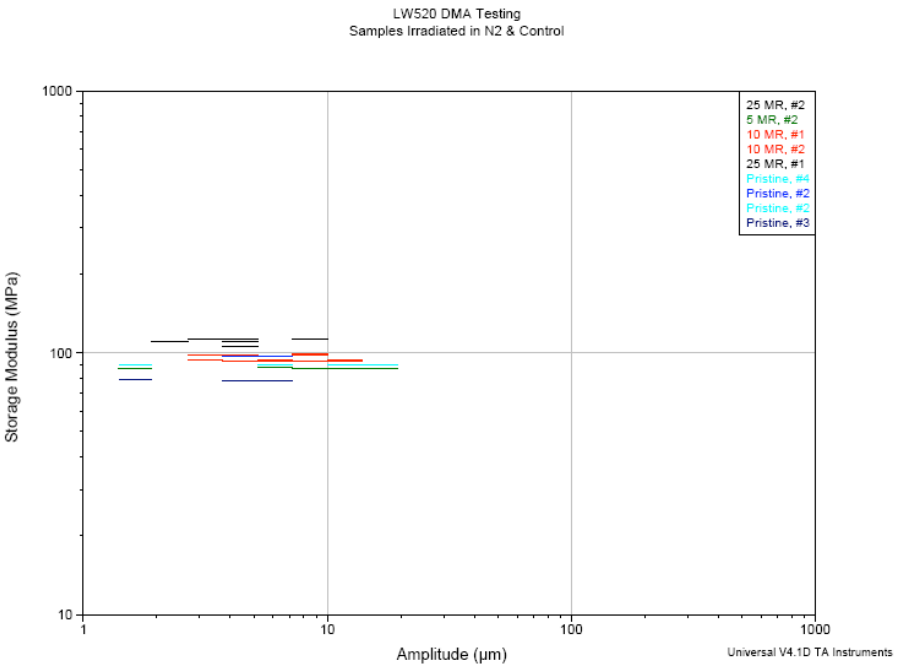

Fig 5. DMA results of adiprene in $\mathrm{N}_{2}$. 
DMA analysis of filled adiprene shows an increase in storage modulus with increasing radiation in nitrogen atmosphere. In contrast to the NMR results, no changes were observed in samples irradiated in air. Though this discrepancy will certainly be investigated, the changes only occur at high doses and at stockpile relevant conditions the adiprene polymer displays great resistance to radiation induced crosslinking.

\subsection{Investigation of S5370 Surveillance Returns:}

Samples of S5370 cushion from different parts were obtained from KCP for IR analyses. It is believed that the unreacted silane present in the silicone couples with $\mathrm{Si}-\mathrm{OH}$ groups in presence of residual tin catalyst [13]. In due course, this additional crosslinking results in performance degradation of the material. In order to establish this, at least 10 samples of S5370 analyzed by ATR-FTIR to look for the presence of the residual silane i.e., the Si-H stretching at $2100 \mathrm{~cm}^{-1}$, shown in Figure 6. However, no evidence was found for the presence of unreacted silane from the IR spectra.

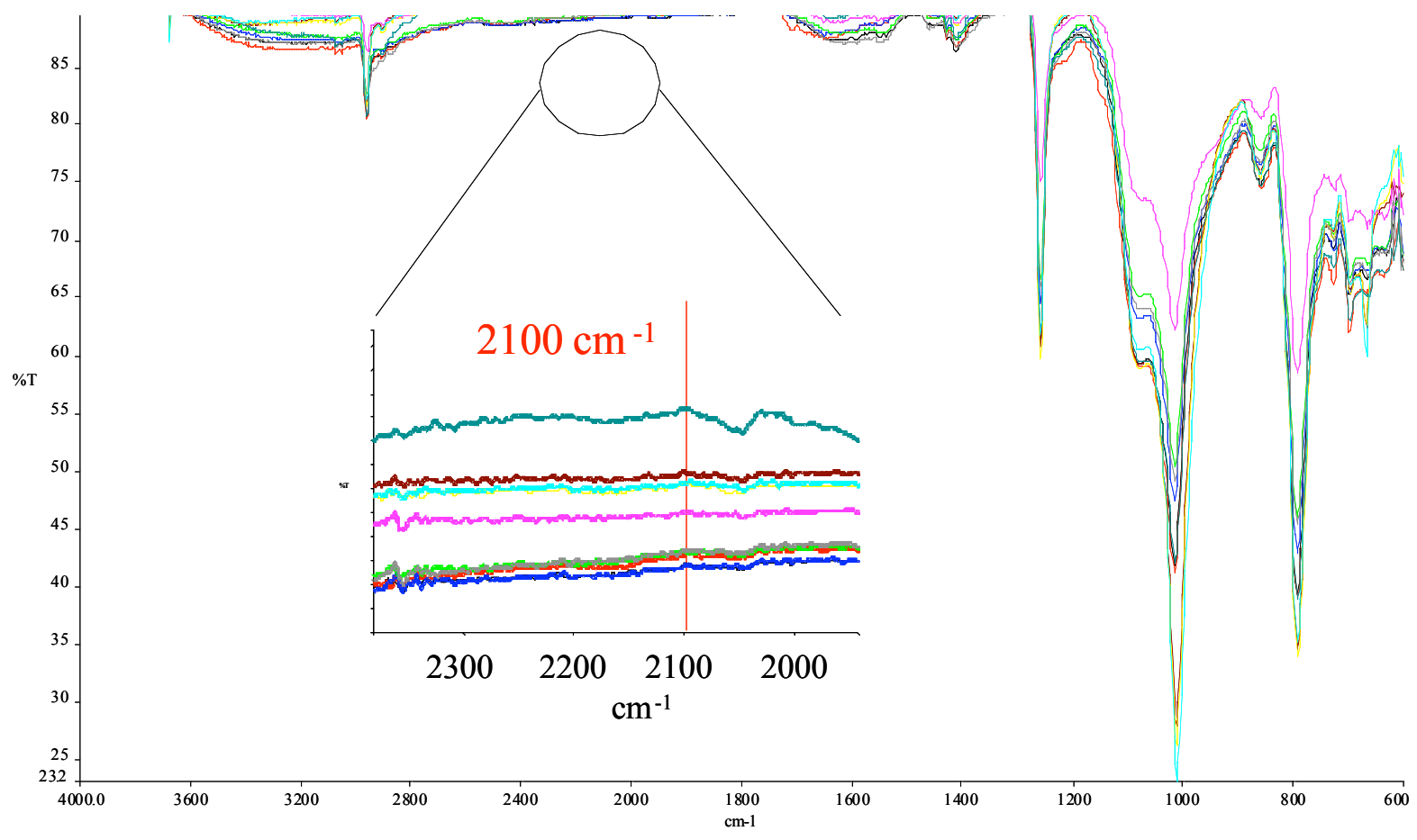

Fig 6. IR spectra of ten surveillance return samples of S5370.

\subsection{Compression set}

A combination of long term stress relaxation experiments are planned to understand long term reduction of load retention in silicon foams. Time-temperature superposition curves (TTS) are being generated to understand the long-term compression behavior of silicone foams. These studies are being done at different temperatures and compressions are aimed at to predict load retention behavior. For short-term tests and lifetime predictions, we plan to study a series of M97xx, TR55, S5370 (high density), and S5455. Initial methods development and testing have been performed on M9747. Long-term testing will be performed on one M97 sample and high 
density S5370. Below are summaries of the short term and long term testing plans and results to date.

\section{Short term testing}

The stress relaxation studies were conducted using a TA Instruments Q800 Dynamic Mechanical Analyzer using circular samples of 3/8" diameter. The experimental method consisted of placing the sample in the clamp and imposing a small normal force $(0.005 \mathrm{~N})$, just enough to ensure that the plates were in contact with the sample. The temperature was then allowed to equilibrate at the test temperature, all the while maintaining a $0.005 \mathrm{~N}$ normal force on the sample. After a twenty-minute isothermal soak at the test temperature, the sample thickness was measured and a $25 \%$ compressive strain was then imposed on the sample. The strain was held constant for 24 hours, all the while measuring the force being imposed on the sample. After the 24 hours had elapsed, the strain was removed (back to a $0.005 \mathrm{~N}$ normal force) and the sample was allowed to rest for 30 minutes while maintaining the test temperature. After the 30 minutes had elapsed, the sample thickness was measured again. The stress-relaxation data are collected at $25^{\circ}, 50^{\circ}, 75^{\circ}, 100^{\circ}, 125^{\circ}, 150^{\circ}$ and $175^{\circ} \mathrm{C}$. It is intended to create a TTS master curve from this data so that we may extrapolate the long-term stress relaxation behavior of a material.

\begin{tabular}{c|c|c|c}
\hline \hline $\begin{array}{c}\text { Temp } \\
\left({ }^{0} \mathbf{C}\right)\end{array}$ & $\begin{array}{c}\text { Relaxation Modulus (Mpa) } \\
\text { @1000 min }\end{array}$ & $\begin{array}{c}\text { Decrease in thickness } \\
\text { (\%) at 25\% strain }\end{array}$ & Recovery (\%) \\
\hline 21 & 0.142 & 3.6 & 96.4 \\
50 & 0.154 & 2.8 & 97.2 \\
75 & 0.154 & 3.2 & 96.8 \\
100 & 0.163 & 3.5 & 96.5 \\
125 & 0.173 & 3.1 & 96.9 \\
150 & 0.172 & 4.8 & 95.2 \\
175 & 0.186 & 4 & 96 \\
\hline \hline
\end{tabular}

Table 1. Results of short-term relaxation studies of M9747.

The M9747 cellular silicone material was processed from vinyl end-capped silicone prepolymers, silica fillers, and urea as the leachable filler using t-butyl per 2-methylbenzoate as the free radical cross-linking agent. As displayed in Table 1, this material exhibits a negative thickness change of around 3-5\% upon the removal of a constant strain of $25 \%$ at various temperatures. In other words, the material recovery to its original dimensions is around $95-97 \%$.

The loss of recovery is due to the combination of physical and chemical relaxations. The physical process involves the rearrangement of entanglements and diffusion of chain ends which have the potential to recover with time. Some of the physical relaxation may be recoverable by using a critical solvent or temperature thereby increasing the energy of activation. The chemical process involves bond scission and formation of cross-links resulting from breakage and formation of covalent bonds. For this reason, the chemical relaxation processes are nonrecoverable.

It is reasonable to assume given the fact that M9747 is vinyl end-capped, during compression a large number of chain ends with vinyl moieties will be generated. This can lead to self-adhesion of chain ends and formation of cross-links. The hydrolytic degradation of Si-O bond due to the presence of trace amounts of water also results in stress relaxation via backbone 
scission. This results in the formation of silanol groups which are then condensed in the relaxed state to form new network. A steady increase of modulus with increase in temperature indicates the combination of stress relaxation via hydrolytic degradation of $\mathrm{Si}-\mathrm{O}-\mathrm{Si}$ back bone and formation of chain end aggregates.

\section{Long-term testing}

In order to provide some data points at longer times to compare with the predictions made using the short-term experiments, long-term stress-relaxation studies of M9763 and S5370HD have been designed. As the reduction in load retention has been observed to occur most rapidly in the first two years of aging, as observed by Schneider [14] and Patel [15], the samples of interested will be tested every month for a total of two years. A test fixture, shown in Figure 7, was designed, which will hold the samples at $30 \%$ compression while aging under constant temperature conditions. Four temperatures will be tested to provide a series of data points for validation of the lifetime

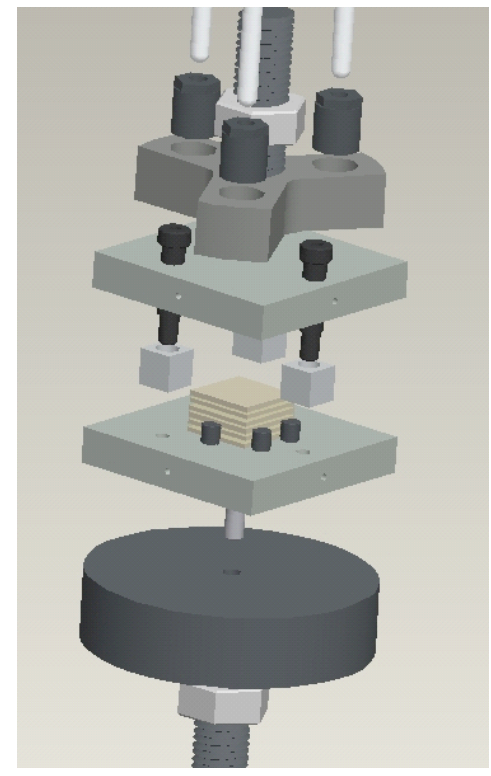

Fig. 7 Design of compression apparatus for long-term testing. predictions from the TTS curves mentioned above. More detailed information can be found in reference [16].

\subsection{Silicone outgassing}

We have significantly improved the accuracy of our $\mathrm{H}_{2} \mathrm{O}$ outgassing model in comparison with last year through the use of the isoconversion technique. We have also performed simple outgassing experiments from thin silicone disks to approximate the magnitude of the diffusion coefficient of $\mathrm{H}_{2} \mathrm{O}$ through M9787 and TR55. The measured diffusion coefficient is on the order of $\sim 2-4 \times 10^{-7} \mathrm{~cm}^{2} \mathrm{~s}^{-1}$ at $293 \mathrm{~K}$. This fast diffusion coefficient suggests
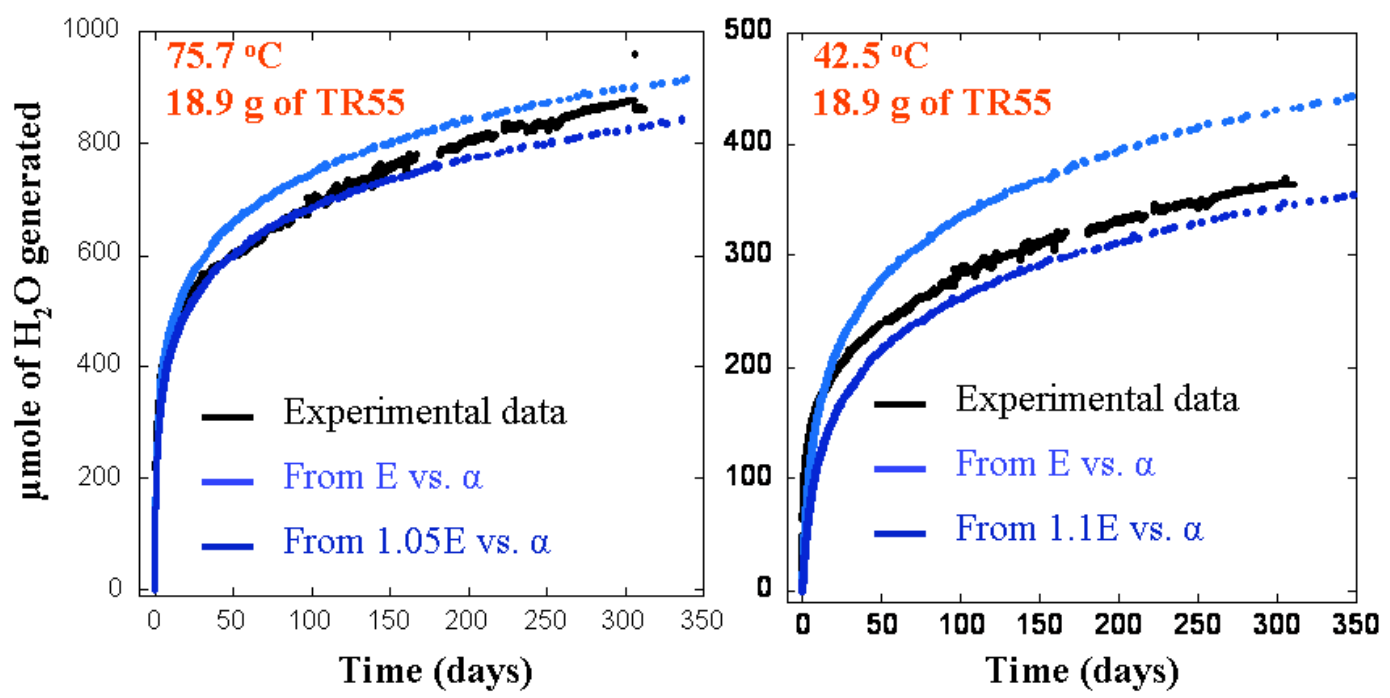

Fig. 8 TR55 $\mathrm{H}_{2} \mathrm{O}$ outgassing models vs. long term isothermal outgassing data 
that transport of $\mathrm{H}_{2} \mathrm{O}$ through silicone can be practically ignored in $\mathrm{H}_{2} \mathrm{O}$ outgassing models since the $\mathrm{H}_{2} \mathrm{O}$ outgassing process happens slowly over many decades.

In Fig. 8, we show the greatly improved agreement between this year TR55 $\mathrm{H}_{2} \mathrm{O}$ outgassing models vs. long term isothermal outgassing data from Y12. Note that the isoconversion technique of thermal analysis mentioned above has resulted in great accuracy for the model even at lower temperature and at shorter time scale. The predicted $\mathrm{H}_{2} \mathrm{O}$ outgassing curves also assume similar shapes as the isothermal $\mathrm{H}_{2} \mathrm{O}$ outgassing curves from Y12. More details of this work can be found in reference [7].

\subsection{DPB getter vapor pressure}

We have measured the equilibrium vapor pressures of virgin and fully-hydrogenated DPB and confirmed that fully-hydrogenated DPB is extremely volatile. A mathematical model to analyze the hydrogen uptake kinetics from microbalance experiment has been formulated to take into account both the geometry of the pellet and the volatility of the product. Microbalance experiments to obtain hydrogen uptake kinetics of DPB are underway.

It can be observed from Fig. 9 that the vapor pressures of the virgin DPB pellets were more scattered and a little lower than those of commercial virgin DPB pellets. The vapor pressures of fully-hydrogenated DPB were many decades higher than those of virgin counterparts. This suggests that hydrogenated DPB is very volatile. More details of this work can be found in reference [8].

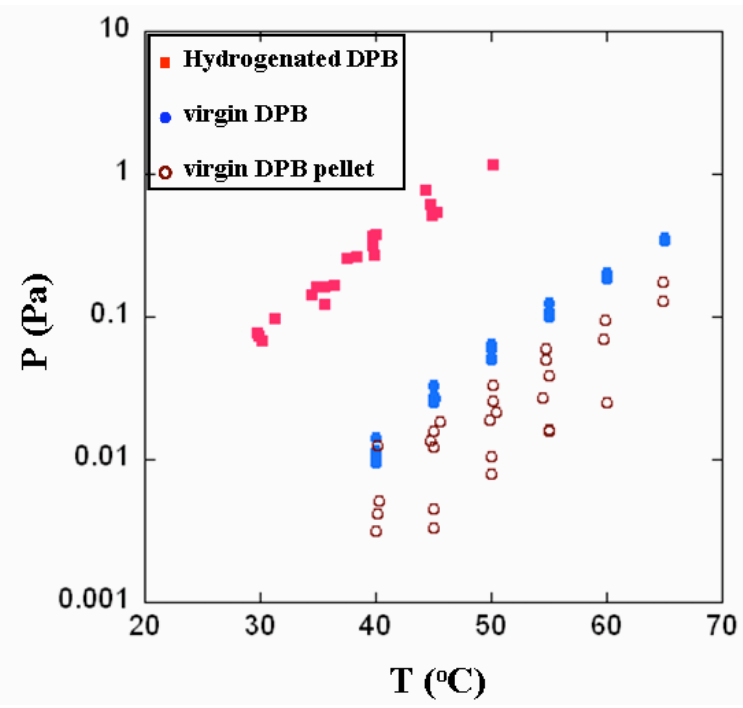

Fig. 9 Vapor pressures of the virgin DPB pellets

\subsection{Other investigations of non-nuclear organic material}

In addition to the work described above, we have performed NMR analysis on other materials including fluoropolymer HE binders and DC745 silicone pads. NMR spectroscopy was been used to investigate the effects of $\gamma$-irradiation in the fluoropolymer FPC-461 [17]. Through a combination of magic angle spinning and variable temperature solid state NMR, significant degradation was seen in the vinyl chloride region of the polymer by ${ }^{1} \mathrm{H} \mathrm{NMR}$, while negligible changes were seen in the chlorotrifluoroethylene block using ${ }^{19} \mathrm{~F} \mathrm{NMR}$. The additional effects of oxygen in the degradation processes in the vinyl chloride region were seen when comparing the samples irradiated in air and nitrogen environments. 
We have used combined use of traditional polymer assessment methods with advanced NMR relaxometry methods to assess changes in the network structure in a series of radiatively aged PDMS based elastomers based on the Dow Corning DC745 gum stock [5]. The NMR data suggests that with increasing exposure, DC745 undergoes radiative crosslinking reactions that, up to $25 \mathrm{MR}$ do not increase significantly the sol fraction of the network. The MQ NMR further suggests a bi-modal network with narrow distributions of residual dipolar couplings. Speciation in the two domains is dose dependent. These methods can also provide improved insight into the degradation of silicone elastomers that leads to permanent set. Our results on DC-745U have quantified the amount of tensile set that occurs in these materials when irradiated under strain [6]. Unilateral NMR relaxometry using the NMR MOUSE and multiple quantum NMR spectroscopy using high field instrumentation were used to quantify the changes in segmental dynamics occurring in these materials and showed that there was an overall reduction in the segmental mobility in samples irradiated with and without tensile strain. However, tensile testing of the damaged and set materials indicated that the materials are softening. It was determined that a reduction in the filler-polymer interaction has been observed in the MQ NMR measurements and given the small decrease in tensile stiffness in these materials it is likely that it is this reduction that is responsible for the softening affect.

In addition to the peer reviewed publications described above, this work has been presented in numerous program meetings [12, 18-23] and internal conferences[24-26].

\subsection{Conclusions:}

This report satisfies the milestones/deliverables for the L3 task "Update chemical and mechanical aging models for cushion/pads/adhesives." We have performed initial aging investigations of UNI620-3 and Adiprene, both of which show complex aging phenomena. The presence of residual silane signatures in S5370 surveillance returns was investigated but no signatures were found. Short and long term stress relaxation experiments are underway on M97 and high density S5370 materials and initial results on M9747 reveal a combination of hydrolytic degradation of the Si-O-Si backbone and the formation of chain end aggregates. Finally, we have used temperature programmed desorption (TPD) studies to significantly improve the accuracy of our $\mathrm{H}_{2} \mathrm{O}$ outgassing model in comparison with last year through the use of the isoconversion technique as well as to measure the equilibrium vapor pressure of hydrogenated DPB pellets. This work has been presented in a variety of peer-review journals, programmatic meetings, and international conferences. All data reported here will be input into L 2 milestones, "Bill's token L2 milesone".

\subsection{References:}

[1] D. Evans and M. A. Crook, Irradiation of plastics: damage and gas evolution. MRS Bulletin, 1997; 22:36.

[2] Gee, et al, Molecular Dynamics Studies on the Effects of Water Speciation on Interfacial Structure and Dynamics in Silica-Filled PDMS Composites MRS preceedings, submitted (2001) UCRL-JC-144790.

[3] Maxwell, et al, Aging of Silica-Filled PDMS/PDPS Copolymers in Desiccating Environments - a DSC and NMR Study, Macromol. Symposia, submitted (2002) UCRLJC-150691. 
[4] Maxwell, R.Balazs, B. Residual dipolar coupling for the assessment of crosslink density changes in $\gamma$-irradiated silica-PDMS composite materials. J. Chem. Phys. 2002; 116: 10492.

[5] Maxwell, R.S., Chinn, S.C., Solyom, D., Cohenour, R. "Radiation induced degradation in a silica-filled silicone elastomer as investigated by Multiple Quantum ${ }^{1} \mathrm{H}$ NMR", Macromolecules 2005; 38: 7026 [UCRL-JRNL-208180].

[6] Chinn, S.C., DeTeresa, S., Shields, A., Sawvel, A., Balazs, B. Maxwell, R.S., "Chemical Origins of Permanent Set in a Peroxide Cured Filled Silicone Elastomer - Tensile and ${ }^{1} \mathrm{H}$ NMR Analysis" Polymer Degradation and Stabilitiy, in press [UCRL-JRNL-207686].

[7] L.N. Dinh, " $\mathrm{H}_{2} \mathrm{O}$ outgassing from and transport in silicones" LLNL report.

[8] L.N. Dinh, "Vapor pressures and hydrogen uptake kinetics of DPB" LLNL report.

[9] K. Andrianov, G. Slonimskii, A. Zhdanov, V. Levin, Y. Godovakii, and V. Moskalenko, J. Poly. Sci. A, 1972; 10: 1.

[10] Maxwell, R.S., Chinn, S.C., Herberg, J.L., Harvey, C., Alviso, C., Vance, A., Cohenour, R., Wilson, M., Solyom, D., "Baseline and Lifetime Assessments for DC745U Elastomeric Components" LLNL Report, 2005, [UCRL-TR-208929].

[11] Hammon, H.G., Hetherington, N., "Properties of Three Adiprene-Based Adhesives" LLNL Report, 1976, UCRL-52044.

[12] Chinn, S.C., Maxwell, R.S., Herberg, J.L., Gjersing, E.L., Sawvel, A.M., "Magnetic Resonance Investigations of Polymeric Foams and Adhesives" JOWOG 28 Main Meeting, Las Vegas, NV, November 2005.

[13] Patel, M., "Problematic Species in Foamed RTV Polysiloxane Rubbers and the Impact on the Useful Properties of the Rubber", DP Foams Workshop, Sandia National Laboratory, January, 2005.

[14] Schneider, J.W., "2 Year Life Test Results for Dow Corning's S-5370, P.O. \#201-0-02” Honeywell Federal Manufacturing \& Technologies Internal Memorandum, December 20, 1982.

[15] Patel, M., Skinner, A.R., "Thermal ageing studies on room-temperature vulcanized polysiloxane rubbers" Poly. Degrad. Stab. 2001; 73:399.

[16] Chinn, S. "S5370 Cushion Test" Internal memo to Tom Healy et al.

[17] Chinn, S.C., Wilson, T.S., Maxwell, R.S., "Multinuclear Analysis of Radiation Induced Degradation in FPC-461 Fluoropolymers by Variable Temperature NMR," Polymer Degradation and Stability, in press [UCRL-JRNL-207685].

[18] Sawvel, A.M., Chinn, S.C., Maxwell, R.S., "NMR Relaxation Studies of Polymeric Foams using the NMR MOUSE", DP Foams Workshop, Sandia National Laboratory, January, 2005 [UCRL-PRES-209585].

[19] Chinn, S.C., Maxwell, R.S., "Multiple Quantum ${ }^{1}$ H NMR for the Analysis of DP Silicone Foams" DP Foams Workshop, Sandia National Laboratory, January 2005 [UCRL-PRES210796].

[20] Chinn, S.C., Sawvel, A.M., Maxwell, R.S., "Benchtop NMR Relaxation Studies of Polymeric Foams and Adhesives" Compatibility, Aging, and Stockpile Stewardship Conference, Savannah River National Lab, Aiken, SC, March 2005, [UCRL-PRES209585].

[21] Herberg, J.L., Gjersing, E.L., Chinn, S.C., Maxwell, R.S. “3D Mapping of Polymer Crosslink Density with Magnetic Resonance Imaging” Compatibility, Aging, and Stockpile 
Stewardship Conference, Savannah River National Lab, Aiken, SC, March 2005, oral presentation [UCRL-PRES-211736].

[22] Chinn, S.C, Sawvel, A.M., Maxwell, R.S., "Solid State NMR Investigations of Aged Polymeric Foams and Adhesives" $34^{\text {th }}$ PolyMAC, Y12 National Security Complex, Oak Ridge, TN, May, 2005, [UCRL-PRES-213730].

[23] Chinn, S.C., Herberg, J.L., Gjersing, E.L., Sawvel, A.M., Maxwell, R.S., "Magnetic Resonance as a Diagnostic Tool for Polymer Characterization" $34^{\text {th }}$ PolyMAC, Y12 National Security Complex, Oak Ridge, TN, May, 2005, oral presentation [UCRL-PRES213731].

[24] Chinn, S.C., Herberg, J.L., Sawvel, A.M., Maxwell, R.S., "Solid State NMR Measurements for Preliminary Lifetime Assessments in $\gamma$-Irradiated and Thermally Aged Siloxane Elastomers" Materials Research Society Fall Meeting, Boston, MA, December 2004 [UCRL-PRES-208548].

[25] Maxwell, R.S., Chinn, S.C., Gee, R.H., Patel. M., Balazs, B., "NMR and MD Approaches to the Study of Interfacial Interactions in the Aging of Filled Silicone Elastomters," $229^{\text {th }}$ National American Chemical Society Meeting, San Diego, CA, March 2005, [UCRLPRES-210499].

[26] Chinn, S.C., Herberg, J.L, Gjersing, E.L., Sawvel, A.M., Maxwell, R.S., "Solid State NMR Measurements for Preliminary Lifetime Assessments in $\gamma$-Irradiated and Thermally Aged Siloxane Elastomers" $44^{\text {th }}$ Experimental NMR Conference, Providence, RI, April 2005, [UCRL-POST-203956]. 